\title{
Le dépérissement observé en Isère sur sapin et épicéa : relations avec les caractéristiques physico-chimiques des écorces
}

\author{
I Legrand 1, J Asta 1 \\ avec la collaboration technique de Y Goudard ${ }^{2}$ \\ 1 Laboratoire de Biologie Alpine, Université Joseph-Fourier, Grenoble BP 53 X, \\ 38041 Grenoble Cedex; \\ 2 ONF, 38000 Grenoble, France
}

(Reçu le 16 septembre 1992; accepté le 11 janvier 1993)

\begin{abstract}
Résumé - Dans le cadre d'une étude sur le dépérissement des forêts dans les Alpes du Nord (massifs de Belledonne, Vercors et Chartreuse), nous avons prélevé des échantillons d'écorce sur le tiers des 474 sapins et 682 épicéas faisant l'objet de notations sur leur état de dépérissement (épaisseur des 3 derniers cernes, pourcentage de perte d'aiguilles et pourcentage de couleur anormale du houppier). Les écorces récoltées ont été analysées en laboratoire $(\mathrm{pH}$, conductivité et épaisseur de l'écorce). Des analyses statistiques ont mis en évidence des différences dans le degré de dépérissement entre sapins et épicéas, ainsi qu'entre massifs : si le massif du Vercors est moins touché par le phénomène, c'est en Belledonne qu'on observe la plus grande proportion d'arbres très dépérissants, le sapin semblant plus atteint que l'épicéa dans ce massif. L'analyse des données sur les écorces apporte des résultats intéressants sur les variations des caractéristiques physicochimiques, aussi bien à l'intérieur d'une même essence qu'entre sapins et épicéas, mais également entre massifs, et nous tentons de donner une explication aux différences observées. L'analyse des relations entre caractéristiques physico-chimiques des écorces et critères de dépérissement montre que les arbres les plus dépérissants ont statistiquement une écorce plus acide et de conductivité plus faible que celle des arbres sains. Le processus d'échange protons-cations au niveau du feuillage pourrait donner une explication à cette relation.
\end{abstract}

dépérissement forestier / sapin (Abies alba Mill) / épicéa (Picea abies (L) Karsten) / Alpes / acidité et conductivité d'écorce

Summary - Forest decline observed in the department of Isère (France) on silver fir trees (Abies alba Mill) and Norway spruce trees (Picea abies (L) Karsten): relationships with the physico-chemical characteristics of the barks. In recent years, the decline of the mountain forests in Europe has been given very serious consideration; this phenomenon has been partly attrib- 
uted to diffuse atmospheric pollution. As it was impossible for us to study the effects of such pollution on trees directly we concentrated on the relationships that might exist between bark physico-chemical characteristics and criteria of tree decline (thickness of the 3 outermost tree rings, percentage of needle loss, percentage of abnormal colour). In 50\% of the trees studied (165 silver firs and 235 Norway spruces), bark samples were taken to measure their $\mathrm{pH}$ and conductivity in the laboratory. Before starting this study, methods had to be defined: an experimental procedure had to be drawn up for bark analysis suited to our objectives. The processing of all data was based on statistical methods.

The study of the criteria of tree decline, including that of bark physico-chemical characteristics, highlighted the following important points:

- the Belledonne massif is more affected by the decline phenomenon and in this massif, silver firs are more affected than Norway spruces (fig 1);

- it was noted that Norway spruce barks are on average more acid than silver fir barks, and that they are also thinner (fig 2);

- the correlations between bark acidity, conductivity and thickness showed that conductivity is closely related to bark thickness, whereas for acidity, there is no significant difference to be observed (table 1);

- differences in acidity, conductivity and bark thickness are to be noted between the various massifs; these differences can be explained (figs 3 and 4);

- the study of the correlations between the criteria of decline and bark characteristics showed that the seriously damaged silver firs had more acid barks in general than healthy trees. Conversely in the Norway spruces, this aspect was not observed possibly because their barks are already naturally more acid (fig 5);

- as far as conductivity is concerned, this appears to be lower in the barks of damaged trees, both silver firs and Norway spruces (fig 5).

It is suggested that the differences observed are not directly due to the deposition of polluting agents on the barks, but that the process of 'recretion' in the leaves could provide an explanation: among damaged trees and hence the most defoliated specimens, rainwater is less charged in cations in contact with the leaves, and as the water runs down the bark there is less protoncation exchange, and the bark remains more acid than in the case of a healthy tree.

forest decline / silver-fir (Abies alba Mill) / Norway spruce (Picea abies (L) Karsten) / Alps / acidity and bark conductivity

\section{INTRODUCTION}

Depuis quelques années, il est fortement question du dépérissement des forêts, aussi bien en France qu'en Europe occidentale (Bouvarel, 1984; Barthod et al, 1987; Bonneau et Landmann, 1988). Constaté dans l'ensemble des massifs montagneux, ce phénomène est aujourd'hui en partie attribué à la pollution atmosphérique diffuse (ozone, acidification), mais d'autres causes interviennent également, telles que les sécheresses répétées de ces dernières années, ainsi que les déséquilibres minéraux enregistrés au ni- veau du sol (Bonneau, 1989, 1990; Landmann, 1991).

Dans le cadre du programme français DEFORPA (dépérissement des forêts attribué à la pollution atmosphérique), programme élaboré dès 1984 pour rechercher les causes réelles du dépérissement actuel des forêts, le laboratoire de Biologie alpine de l'université Joseph-Fourier a présenté fin 1986 un projet de caractérisation des formes du dépérissement affectant les sapins et les épicéas dans les massifs dauphinois externes, en collaboration avec I'Office national des forêts de l'Isère (Souchier, 1989). 
Si la pollution est réellement une des causes de la perte de vitalité des arbres, on pouvait se demander si les lichens, organismes très sensibles à divers polluants bien connus (tels que le dioxyde de soufre, le plomb, le fluor, etc) (Asta, 1980; Belandria, 1986; Deruelle et Lallemant, 1983), subissaient également les atteintes de la pollution diffuse, soit directement par l'intermédiaire de l'atmosphère dont ils dépendent entièrement pour leurs échanges nutritifs, soit indirectement par l'éventuelle modification physico-chimique de l'écorce qui leur sert de support (Härtel et Grill, 1972; Härtel, 1982; Lötschert et Köhm, 1977). Le programme DEFORPA élaboré en Isère nous a donné l'occasion de tester sur un échantillonnage très important les relations qui pouvaient exister entre l'état de dépérissement des arbres et les lichens, d'une part, l'acidité et la conductivité des écorces, d'autre part. Les résultats relatifs aux lichens sont exposés par ailleurs (Legrand et Asta, 1991). Nous présentons ici les résultats concernant l'étude sur les relations entre les caractéristiques des écorces et les critères de dépérissement des arbres. Précisons également que le terme d'aécorce» utilisé dans le texte désigne la partie prélevée, c'est-à-dire le rhytidome.

\section{MATÉRIEL ET MÉTHODES}

\section{Travail de terrain}

Cent soixante-deux placettes forestières choisies à partir du fichier de I'Inventaire forestier national ont pu être étudiées dans l'étage montagnard des massifs de Belledonne, Chartreuse et Vercors (Isère, Alpes du Nord), au cours de l'étè 1987. L'ensemble réunissait 474 sapins et 682 épicéas. Sur chacune de ces placettes, qui correspondent à des surfaces concentriques de $15 \mathrm{~m}$ de diamètre, différentes notations ont été effectuées, pour préciser les caractéristiques suivantes:
- caractéristiques stationnelles : altitude, exposition, topographie, microrelief, roche-mère, sols et humus;

- végétation et peuplement : traitement forestier, essence principale, relevés phytosociologiques (dont les lichens) quantifiés par strate;

- critère de symptomatologie: sur les quelques 25 paramètres établis par les forestiers et relevés sur le terrain, nous ne mentionnons que ceux qui ont présenté un intérêt remarquable après traitement des données : accroissement des cernes des 3 dernières années $(0-3,4-5,6-$ $7,8-45 \mathrm{~mm}$ ); pourcentage de perte d'aiguilles $(0,5-10,15-30$, et plus de $35 \%)$; pourcentage de couleur anormale du houppier $(0, \geq 0-25, \geq 25$ $60 \%)$;

- prélèvements d'écorce : le prélèvement d'écorce a èté réalisé sur le tiers des arbres, l'échantillonnage ayant été fait en retenant des placettes au hasard. Des cylindres d'écorces ont donc été prélevés sur 165 sapins et 235 épicéas, à une hauteur de $1,50 \mathrm{~m}$, à l'aide d'un marteau et d'un emporte-pièce $(2 \mathrm{~cm}$ de diamètre).

\section{Travail de laboratoire}

La méthode présentée ici a été mise au point par les auteurs (Legrand, 1991; Legrand et Asta, 1991).

Les morceaux d'écorces sont d'abord brossés, puis les cylindres sont coupés au couteau pour en prélever la partie externe (2 à $3 \mathrm{~mm}$ ). Chaque échantillon est ensuite mis à macérer $24 \mathrm{~h}$ au réfrigérateur dans $5 \mathrm{ml}$ d'eau distillée dégazéifiée. Les mesures de $\mathrm{pH}$ sont réalisées à l'aide d'un titrateur (Tacussel type $\Pi T$ processeur 2) à électrode de contact combinée. La conductivité est mesurée avec un résistivimètre à lecture numérique (type CD 60 à électrode type TE 100).

\section{Traitement des données}

L'ensemble des données recueillies a été traité statistiquement. Nous avons utilisé le test de Mann et Whitney, test non paramétrique qui permet de comparer des moyennes 2 à 2, le test de $\chi^{2}$, qui permet de vérifier si la répartition d'une 
population est homogène en fonction des paramètres étudiés et, enfin, des études de corrélation.

\section{RÉSULTATS}

\section{Comparaison du degré de dépérissement observé dans les 3 massifs}

Sur les 383 arbres dont l'écorce a été prélevée et pour lesquels les données sur le pourcentage de perte d'aiguilles existent, c'est en Vercors que la proportion d'arbres sains est la plus élevée, avec $70 \%$ d'arbres ayant moins de $15 \%$ de perte d'aiguilles ( $42 \%$ en Belledonne et $41 \%$ en Chartreuse) (fig 1). Tandis que pour les arbres très dépérissants, Belledonne est le massif le plus atteint avec $14 \%$ des arbres ayant plus de $35 \%$ de perte d'aiguilles ( $10 \%$ en Vercors et autant en Chartreuse). Dans ce massif (Belledonne), le sapin semble plus atteint par les fortes défoliations que l'épicéa : $19 \%$ de sapins ont plus de $35 \%$ de perte d'aiguilles et seulement $11 \%$ d'épicéas; tandis qu'en Vercors, seuls

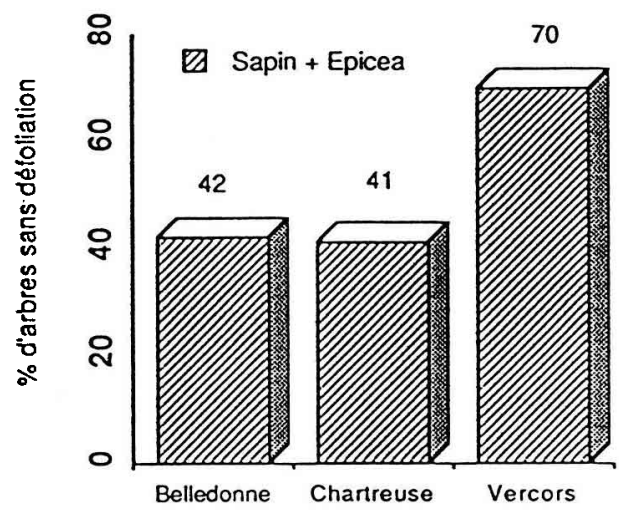

Fig 1. Répartition par massif (Belledonne, Chartreuse, Vercors) des arbres (sapins + épicéas en pourcentages) sans défoliation apparente (0 à 10\% de perte d'aiguilles). les épicéas sont atteints par les fortes défoliations.

Les résultats de la typologie (Souchier, 1989) ont montré que le dépérissement était plutôt associé au type de sol et particulièrement au caractère superficiel du sol pour l'épicéa.

\section{Description des caractéristiques physico-chimiques des écorces}

Avant d'étudier les relations entre le niveau de dépérissement et les caractéristiques des écorces, il est nécessaire de décrire la forme des distributions de l'acidité, de la conductivité et de l'épaisseur des écorces pour le sapin et l'épicéa, de tester les relations qui peuvent exister entre ces $3 \mathrm{cri}$ tères et de comparer les données entre les 3 massifs.

\section{Analyse des distributions (fig 2)}

Si les distributions de l'acidité (partie superficielle de l'écorce) et de l'épaisseur de l'écorce (mesurée jusqu'au cambium) s'apparentent à une loi normale, celle de la conductivité présente un diagramme de type asymétrique.

Sur l'ensemble des arbres observés, l'acidité de l'écorce (fig 2A) varie entre 3,4 et 5,4 unité $\mathrm{pH}$ pour le sapin, et entre 3,2 et 5,2 pour l'épicéa, et en moyenne (moyenne géométrique) l'épicéa a une écorce légèrement plus acide $(4,2)$ que celle du sapin $(4,6)$.

La distribution de la conductivité (fig 2B) est plus étalée pour le sapin (de 25 à 525 $\mu \mathrm{S})$ que pour l'épicéa (de 25 à $425 \mu \mathrm{S}$ ), alors que les moyennes géométriques sont identiques (122 $\mu \mathrm{S})$.

Quant à l'épaisseur de l'écorce (fig 2C), la comparaison des 2 diagrammes indique que le sapin a une écorce plus épaisse 
A
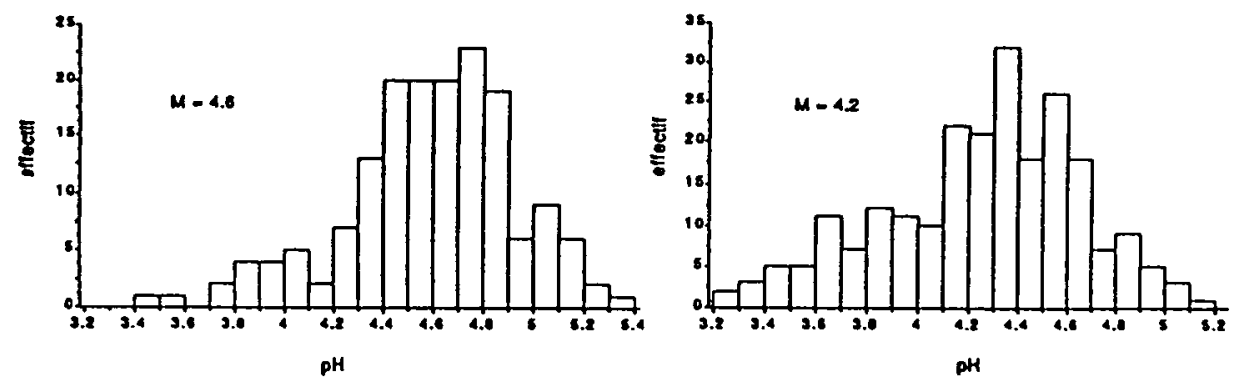

B
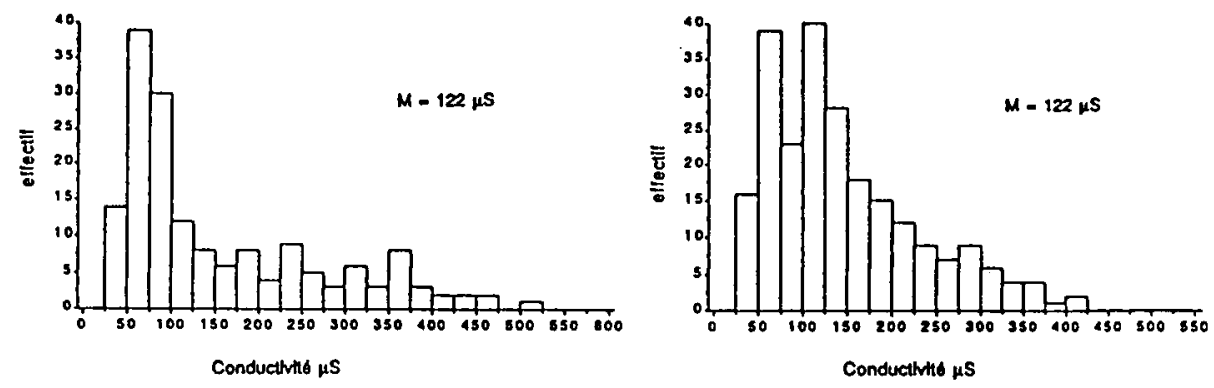

C
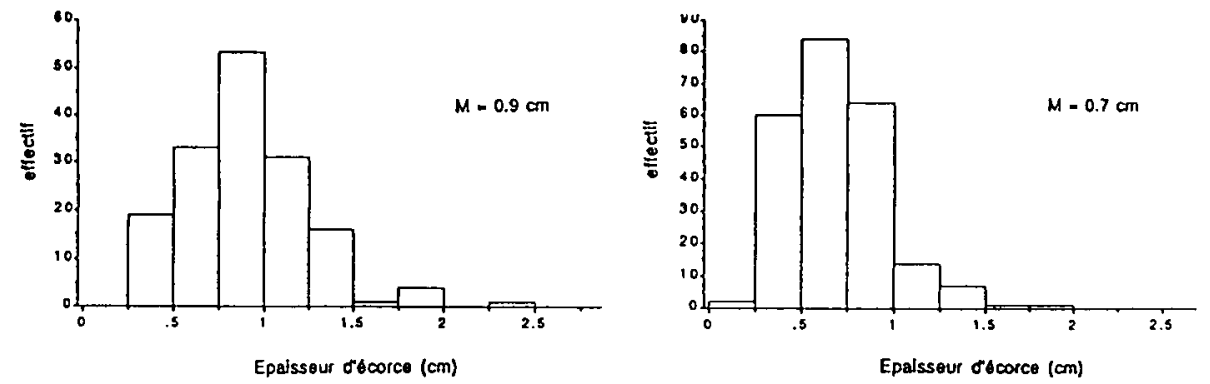

Fig 2. Histogrammes de fréquence des caractéristiques physico-chimiques des écorces pour le sapin (165 individus) et pour l'épicéa (235 individus). A : acidité; B : conductivité; $\mathrm{C}$ : l'épaisseur de l'écorce jusqu'au cambium.

(entre 0,2 et $2,5 \mathrm{~cm}$, moyenne $=0,9$ ) que celle de l'épicéa (entre 0,1 et $2,0 \mathrm{~cm}$, moyenne $=0,7$ ), ce qui peut s'expliquer par le fonctionnement des rhytidomes, dif- férent pour ces 2 espèces : chez l'épicéa, l'écorce s'exfolie par écailles, régulièrement et rapidement, ce qui entraîne une écorce en moyenne peu épaisse, tandis 
que chez le sapin l'écorce reste plus épaisse.

\section{Relations entre acidité, conductivité et épaisseur des écorces}

Pour compléter l'analyse des distributions, il est important de rechercher s'il existe des relations entre l'acidité, la conductivité et l'épaisseur de l'écorce, et d'essayer de comprendre les différences qui se dessinent entre sapins et épicéas. Nous avons donc effectué des études de corrélations entre la conductivité et l'épaisseur de l'écorce, puis entre le $\mathrm{pH}$ et l'épaisseur par essence et par massif (tableau I).

Les corrélations entre la conductivité et l'épaisseur de l'écorce sont toutes significatives, quels que soient le massif et l'essence d'arbre (plus l'épaisseur de l'écorce est importante et plus la conductivité est faible). En revanche, l'acidité n'est pas liée, dans l'ensemble, à l'épaisseur de l'écorce.

Le fait que le $\mathrm{pH}$ ne soit pas ou peu corrélé à l'épaisseur de l'écorce nous fait penser qu'il est dépendant du milieu extérieur. En effet, dans cette étude, il s'agit bien d'avoir un seul couple de données par arbre (à $1,50 \mathrm{~m}$ ) pour presque 400 individus (sapins et épicéas). Or les arbres n'ont pas tous le même âge, donc pas tous la même épaisseur d'écorce à 1,50 m. Et si une modification du milieu survient (pollution atmosphérique ou ruissellement le long du tronc), l'acidité de l'écorce résultante est alors déterminée par le degré de cette perturbation et n'est pas liée à l'épaisseur de l'écorce. Nous reviendrons sur ce point dans la discussion.

En revanche, les excellentes corrélations observées entre conductivité et épaisseur d'écorce indiquent que la conductivité de l'écorce n'est pas liée à un facteur externe, du moins dans les régions étudiées. On peut penser qu'un déterminisme interne masque éventuellement l'effet d'une contamination extérieure.

\section{Comparaison entre les massifs (fig 3)}

La description des données par massif fait apparaître que les sapins et épicéas de Chartreuse ont une écorce plus épaisse que les arbres des autres massifs (fig $3 A$ ), ce qui s'explique par le diamètre moyen plus élevé de la population des arbres en Chartreuse.

Quant aux mesures de conductivité (fig 3B), c'est en Chartreuse qu'elles sont les plus faibles, ce qui s'explique par

Tableau I. Corrélations entre le logarithme népérien $\ln (\mathrm{pH}$ ) et In(épaisseur d'écorce) (noté $\mathrm{pH} / \mathrm{Ep}$ ), et entre In(conductivité) et In(épaisseur) (noté $\mathrm{C} / \mathrm{Ep}$ ), par essence et par massif. Sont indiqués l'effectif (N) ainsi que le seuil des valeurs significatives (R significatif), $\alpha=0,05$. Les valeurs significatives sont soulignées.

\begin{tabular}{|c|c|c|c|c|c|c|c|c|}
\hline & \multicolumn{4}{|c|}{ Sapin } & \multicolumn{4}{|c|}{ Épicéa } \\
\hline & $N$ & R sign & $p H / E p$ & $C / E p$ & $N$ & $R$ sign & $p H / E p$ & $C / E p$ \\
\hline Chartreuse & 61 & 0,25 & $-0,05$ & -0.66 & 60 & 0,25 & -0.32 & -0.31 \\
\hline Vercors & 29 & 0,35 & +0.41 & -0.58 & 54 & 0,26 & $-0,20$ & -0.34 \\
\hline Belledonne & 68 & 0,23 & $-0,22$ & -0.60 & 119 & 0,17 & $+0,07$ & -0.42 \\
\hline
\end{tabular}


A

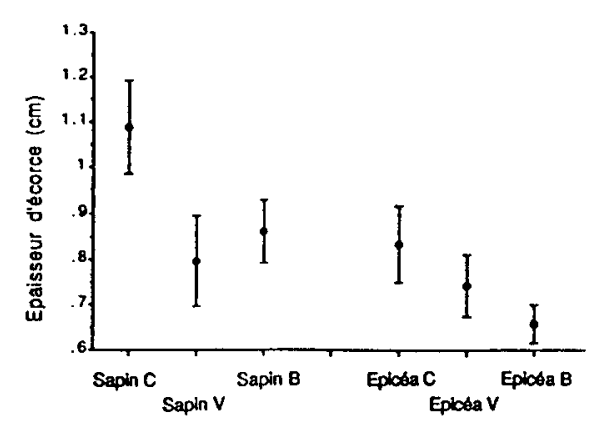

B

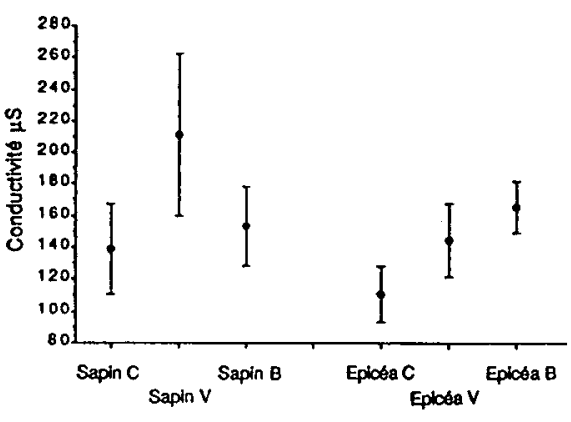

C

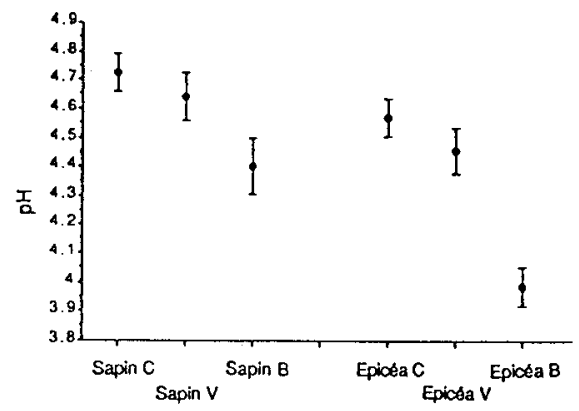

Fig 3. Comparaison des mesures d'épaisseur d'écorce (A) de conductivité (B) et d'acidité (C) pour le sapin et pour l'épicéa par massif $(C=$ Chartreuse, $\mathrm{V}=$ Vercors, $\mathrm{B}=$ Belledonne); (moyenne, intervalle de confiance : $\alpha=0,05$ ). l'épaisseur d'écorce plus importante, relation que nous venons de souligner. En Vercors, la conductivité moyenne des écorces de sapin est la plus élevée, et correspond aux épaisseurs d'écorce les plus faibles.

Si l'on compare les données pH sur l'ensemble des arbres (fig $3 \mathrm{C}$ ), on s'aperçoit que, dans le massif de Belledonne, les arbres ont une écorce plus acide. On peut se demander si cette acidité est due à un niveau de pollution plus acide dans ce massif, ou au fait que la proportion d'arbres très dépérissants y est plus importante. Nous avons donc comparé l'acidité moyenne des écorces par massif, uniquement sur la population des arbres sains, c'est-à-dire présentant de 0 à $5 \%$ de perte d'aiguilles (fig 4). Sur cette figure, nous constatons que, pour les arbres sains, c'est également dans le massif de Belledonne que les écorces sont en moyenne plus acides, que ce soit pour le sapin ou pour l'épicéa. Le dépérissement n'intervient donc pas dans le fait qu'en Belledonne les arbres ont une écorce plus acide. Nous reviendrons également sur ce point dans la discussion.

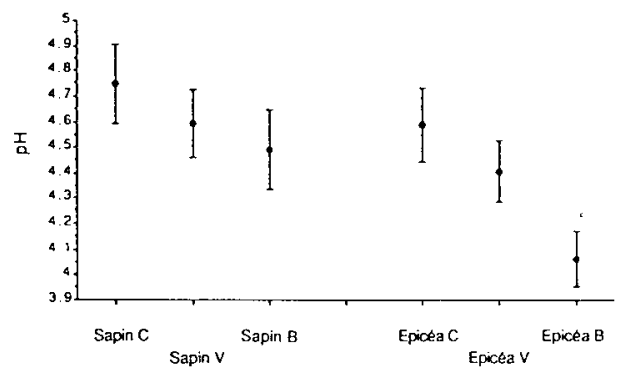

Fig 4. Comparaison des mesures d'acidité d'écorce sur la population des arbres sains (sapins et épicéas ayant moins de $10 \%$ de perte d'aiguilles) par massif ( $C=$ Chartreuse, $V=$ Vercors, $\mathrm{B}=$ Belledonne); (moyenne, intervalle de confiance : $\alpha=0,05$ ). 

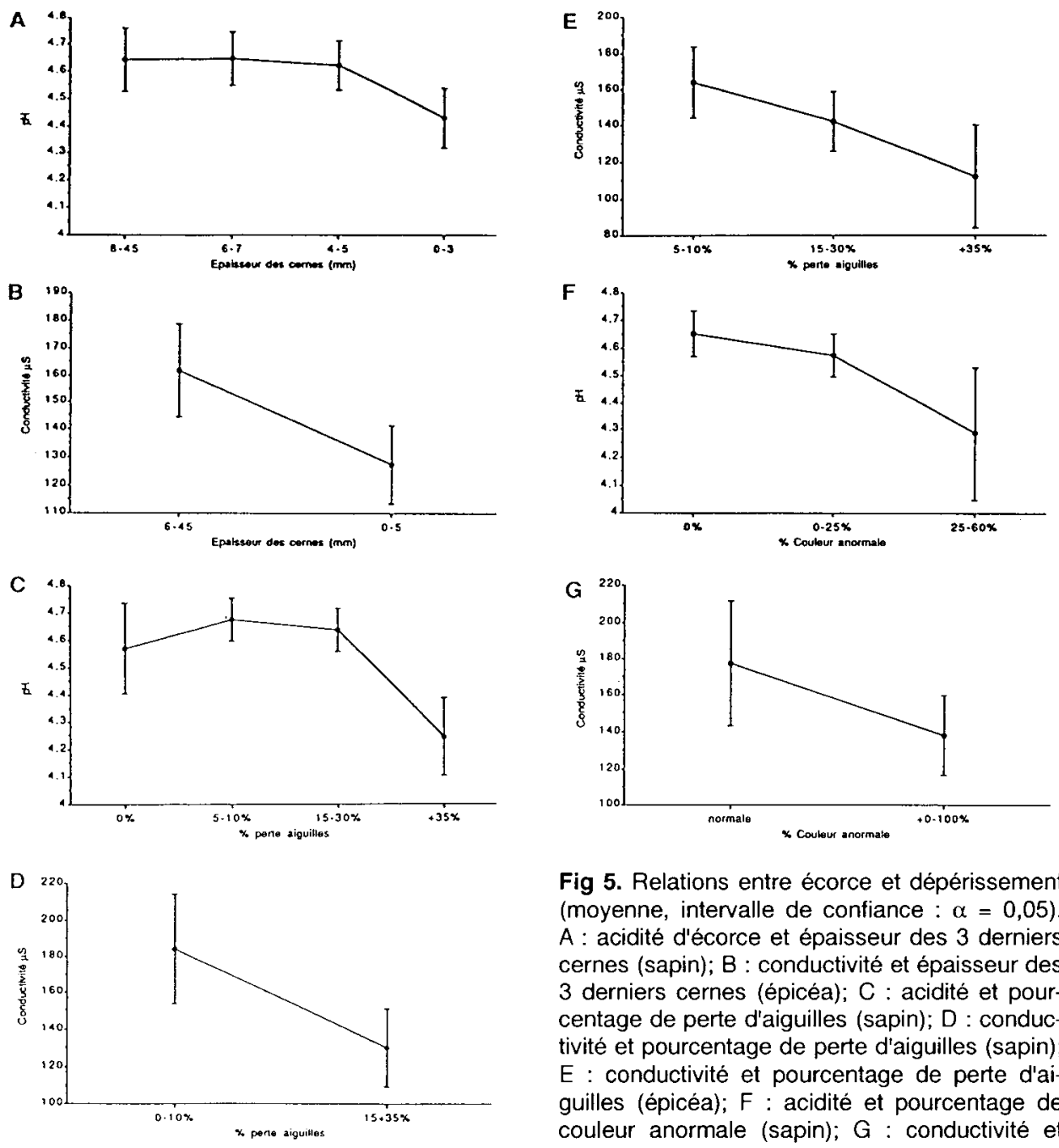

Fig 5. Relations entre écorce et dépérissement (moyenne, intervalle de confiance : $\alpha=0,05$ ). $A$ : acidité d'écorce et épaisseur des 3 derniers cernes (sapin); B : conductivité et épaisseur des 3 derniers cernes (épicéa); $C$ : acidité et pourcentage de perte d'aiguilles (sapin); D : conductivité et pourcentage de perte d'aiguilles (sapin); $E$ : conductivité et pourcentage de perte d'aiguilles (épicéa); $F$ : acidité et pourcentage de couleur anormale (sapin); $G$ : conductivité el pourcentage de couleur anormale (sapin).

\section{Relations entre écorces et dépérissement (fig 5)}

Pour cette étude, le faible échantillonnage de certaines classes peu représentées ne nous a pas permis de séparer les 3 massifs. C'est donc sur la totalité des arbres, par essence, que nous avons appliqué le

test de Mann et Whitney pour vérifier ou non les relations entre les caractéristiques de l'écorce et les effets du dépérissement.

\section{Épaisseur des 3 derniers cernes}

Chez le sapin (fig 5A), l'épaisseur des 3 derniers cernes est significativement liée à 
l'acidité de l'écorce : le $\mathrm{pH}$ diminue quand l'épaisseur des cernes diminue (les cernes les plus minces correspondent à des arbres plutôt dépérissants dont la croissance est faible), ce qui signifie que l'acidité augmente avec le dépérissement. Pour l'épicéa, on n'observe pas de différence significative.

Quant à la conductivité de l'écorce, elle ne dépend du facteur croissance que pour l'épicéa (fig 5B), les cernes les moins épais correspondant à une conductivité plus faible.

\section{Pourcentage de perte d'aiguilles}

Sur sapin (fig $5 \mathrm{C}$ ), les arbres très défoliés ont une écorce plus acide, alors que sur épicéa, on n'observe pas de différence significative.

Quant à la conductivité, les arbres les plus défoliés (sapins, fig 5D, et épicéas, fig $5 \mathrm{E}$ ) ont une conductivité d'écorce plus faible.

\section{Pourcentage de couleur anormale du houppier}

Les caractéristiques physico-chimiques des écorces d'épicéas ne semblent pas être liées au pourcentage de couleur anormale du houppier, alors que pour le sapin, les arbres présentant au moins $25 \%$ de couleur anormale ont une écorce plus acide (fig $5 \mathrm{~F}$ ) et de conductivité plus faible (fig $5 \mathrm{G}$ ) que celle des arbres sains.

\section{DISCUSSION}

L'article présenté ici s'inscrit dans un travail de recherche plus général (Legrand, 1991) dont l'objectif principal était de définir si les lichens corticoles pouvaient servir de bio-indicateurs du dépérissement des arbres, soit en étant directement sensibles aux effets d'une pollution atmosphérique diffuse, soit en réagissant indirectement aux modifications physico-chimiques des écorces qui leur servent de support, l'écorce pouvant être considérée comme l'interface entre l'arbre et le lichen. Les résultats de ce travail ont montré que, contrairement à toute attente, les écorces se sont révélées bien plus riches d'informations que les lichens dans l'étude de ce type de pollution dite diffuse.

Indépendamment du problème du dépérissement, le nombre important d'arbres sondés nous a donné la possibilité d'approfondir les connaissances fondamentales sur cette partie de l'arbre peu étudiée qu'est l'écorce. Nous avons donc constaté des différences remarquables entre sapins et épicéas, mais également entre massifs.

Nous confirmons que le sapin a une écorce plus épaisse que celle de l'épicéa (ce qui peut s'expliquer par le fonctionnement différent des rhytidomes) et que l'écorce des sapins est moins acide. Nous mettons, en revanche, en évidence pour la première fois que la conductivité de la partie superficielle de l'écorce est liée à l'épaisseur de l'écorce, contrairement à l'acidité qui ne lui est pas corrélée. Nous pensons que la conductivité de la partie externe de l'écorce est directement influencée par la proximité des tissus internes riches en ions (liber, cambium), tandis que les variations de l'acidité dépendent du milieu extérieur, dans les conditions stationnelles de notre étude.

Cependant, en Belledonne, nous avons constaté que les écorces étaient plus acides que dans les autres massifs. Or nous avons vu précédemment que ce phénomène ne semble pas lié au dépérissement puisque lorsqu'on compare les populations d'arbres sains des 3 massifs, c'est encore en Belledonne que les écorces sont les plus acides. II y a donc bien un effet lié au facteur massif, qui pourrait s'ex- 
pliquer soit par l'intermédiaire du sol, soit par l'intermédiaire de l'atmosphère.

On peut penser qu'en Belledonne, seul massif cristallin (Vercors et Chartreuse sont calcaires), le sol étant moins riche en calcium et divers cations, la minéralomasse des écorces pourrait être moins importante que pour les arbres des massifs calcaires et, par conséquent, entraîner une plus grande acidité des écorces. Des analyses comparatives de minéralomasse des écorces entre les 3 massifs, complétées par des mesures de $\mathrm{pH}$ et de conductivité, pourraient apporter un élément de réponse. Actuellement, les seules données dont nous disposons concernent les aiguilles; il n'y a pas de différence fondamentale dans les résultats d'analyses foliaires effectuées en Chartreuse et Belledonne sur sapin (Puech, 1991); toutefois, ces analyses ont été réalisées sur un échantillonnage très réduit.

La plus forte acidité des écorces rencontrée en Belledonne pourrait également s'expliquer par l'action directe des poussières arrachèes au sol qui sont piégées par les écorces; celles du massif cristallin pourraient être relativement inertes, tandis qu'on connaît l'action neutralisante des poussières calcaires sur les substrats où elles se déposent.

On peut également envisager qu'en Belledonne il existe une pollution atmosphérique acide plus importante que dans les autres massifs, mais qui n'aurait pas d'effet sur le dépérissement. Par ailleurs, les résultats de la typologie (Souchier, 1989) ont également montré que le dépérissement était plutôt associé au type de sol. De par sa situation géographique, le massif de Belledonne semble plus exposé à la pollution liée aux activités humaines de l'agglomération grenobloise que les autres massifs. II serait intéressant d'installer des capteurs pour analyser les dépôts d'origine atmosphérique, mais un tel protocole n'est pas envisageable actuellement.
En ce qui concerne le dépérissement, nous pouvons dire qu'il y a une relation entre ce dernier et les caractéristiques physico-chimiques des écorces. Pour le sapin, on constate une acidification de l'écorce chez les arbres dépérissants, alors que chez l'épicéa, ce phénomène n'est pas aussi net, peut-être parce que son écorce est déjà naturellement plus acide. Quant à la conductivité, elle semble moins élevée sur les arbres dépérissants, pour les 2 essences.

Comment expliquer tout d'abord l'origine des variations de l'acidité des écorces en fonction du dépérissement?

On pourrait envisager l'intervention directe de la pollution atmosphérique (dépôts secs ou pluie incidente sur le tronc). Or, sur une même placette, les caractéristiques des écorces peuvent être très variables d'un arbre à l'autre, alors que ces arbres sont soumis aux mêmes conditions.

On pourrait également avancer l'hypothèse du lavage des aérosols acides déposés sur les aiguilles avant la pluie. Or plus un arbre est dépérissant, moins il a de feuilles et moins il y a possibilité de lessivage d'aérosols acides, ce qui est en contradiction avec la corrélation négative dépérissement-pH mise en évidence dans cette étude.

Une troisième proposition semble, en revanche intéressante à retenir : celle de l'échange protons-cations : quand la pluie (acide) ruisselle sur les aiguilles, l'eau se charge en cations en cédant des protons (les feuilles sont riches en $\mathrm{K}^{+}$et $\mathrm{Ca}^{++}$). L'eau ainsi enrichie ruisselle le long du tronc et cède alors à l'écorce ces ions $\mathrm{K}^{+}$ et $\mathrm{Ca}^{++}$(la partie superficielle de l'écorce est beaucoup moins riche en cations que les feuilles); nous avons effectivement mis en évidence dans une autre étude (Legrand, 1991) une variation de l'acidité des écorces le long du tronc, le sommet étant moins acide que la base du tronc. Donc, 
plus un arbre est dépérissant, moins il a d'aiguilles, moins les pluviolessivats sont riches en cations et plus l'écorce est acide. Cette hypothèse expliquerait également pourquoi l'écorce d'épicéa est plus acide que celle du sapin et pourquoi la corrélation dépérissement-pH d'écorce est moins bonne pour l'épicéa que pour le sapin. En effet, le port des branches est différent pour ces 2 espèces : dans le cas du sapin, l'eau de ruissellement est plutôt ramenée sur le tronc, alors que chez l'épicéa, l'eau s'en éloigne. II serait intéressant de mettre en place un protocole permettant de comparer la quantité et la teneur en cations des pluviolessivats, à différentes hauteurs le long du tronc, en relation avec la minéralomasse et les caractéristiques physicochimiques des écorces, pour le sapin et pour l'épicéa.

Que ce soit sur sapin ou sur épicéa, les arbres les plus défoliés ont une conductivité d'écorce plus faible. L'hypothèse avancée précédemment (influence des pluviolessivats plus ou moins enrichis en cations) pourrait en partie expliquer cette relation. D'autre part, nous avons obtenu d'excellentes corrélations entre épaisseur d'écorce et conductivité. II y aurait donc une relation entre les tissus vivants internes de l'écorce et la conductivité. D'après Garrec et al (1989), il y a une relation entre la résistance électrique du cambium et la vitalité de l'arbre (la résistance est l'inverse de la conductance) : plus la vitalité diminue et plus la résistance augmente, la résistance dépendant essentiellement des ions mobiles $\mathrm{K}^{+}$. Or la mesure de la résistance électrique du cambium peut être considérée comme une indication de l'activité cambiale de l'arbre, activité qui reflète le fonctionnement du houppier (photosynthèse, transpiration, etc) et du système racinaire (nutrition minérale, alimentation hydrique...). II apparaît donc clair que plus un arbre est dépérissant, plus l'activité cambiale est faible et plus la conductivité de son écorce est également faible. La conductivité de l'écorce dépendrait donc de 2 effets conjugués : un effet externe dû aux pluviolessivats et un effet interne dû à l'activité cambiale, tous deux liés à l'état sanitaire de l'arbre.

\section{CONCLUSION}

Les résultats obtenus permettent de dire que l'écorce se révèle donc être un excellent bio-indicateur du niveau de dépérissement des arbres. Cependant, il est nécessaire de prendre les précautions méthodologiques qui s'imposent. II apparaît que le sapin est plus adapté que l'épicéa à la détection de la pollution diffuse. En effet, la partie externe de l'écorce de sapin étant naturellement moins acide et plus stable que celle de l'épicéa, elle est capable de traduire une évolution à plus long terme. D'autre part, les mesures d'acidité nous semblent plus adaptées que celles de conductivité dans les études sur le dépérissement.

Ce travail a soulevé plusieurs points qui mériteraient d'être approfondis :

- l'origine de l'effet massif constaté sur l'acidité des écorces;

- le rôle des tissus vivants de l'écorce interne sur les caractéristiques physicochimiques des écorces;

- l'influence de l'eau de ruissellement le long du tronc sur ces mêmes caractéristiques.

Nous manquons également de références sur les données d'un arbre situé à l'abri de toute contamination $(\mathrm{pH}$ et conductivité). Cela nécessiterait des études en zone non polluée, dont les résultats apporteraient des données de référence pour des comparaisons avec les études sur la pollution. 


\section{RÉFÉRENCES}

Asta J (1980) Flore et végétation lichéniques des Alpes nord-occidentales : écologie, biogéographie, écophysiologie, biodétection de la pollution fluorée. Thèse d'État, Sciences, Université de Grenoble, $250 p$

Barthod C, Bonneau M, Muller M (1987) Le dépérissement des forêts en Europe tempérée. Universalia 1987. Encyclopaedia Universalis, 233-237

Belandria G (1986) Lichens et pollution atmosphérique dans la région Rhônes-Alpes. Biodétection de la pollution acide et fluorée. Effets des polluants sur la germination des spores. Thèse d'Université mention Écologie, Grenoble, $177 \mathrm{p}$

Bonneau M (1989) Que sait-on maintenant des causes du «dépérissement" des forêts ? Rev For Fr 41 (5), 367-385

Bonneau M (1990) Dépérissement des forêts : sécheresse, pollution acide, autres causes. Sécheresse 1 (1), 44-54

Bonneau M, Landmann G (1988) Le dépérissement des forêts en Europe. La Recherche 205, 1542-1556

Bouvarel P (1984) Le dépérissement des forêts attribué aux dépôts atmosphériques acides. Rev For Fr 3, 173-179

Deruelle S, Lallemant R (1983) Les Lichens témoins de la pollution. Thèmes Vuivert, Université Biologie, $108 p$

Garrec JP, Heymes C, Rose C, De Legge R, Toulemonde JC (1989) Étude de la résistance électrique du cambium dans les épicéas (Picea abies L) dépérissants. Ann Sci For 46, 373-385
Härtel $O$ (1982) Pollutants accumulation by bark. In: Monitoring of air pollutants by plants (Steubing L, Jäger HJ, eds) DR W Junk, The Hague 137-147

Härtel O, Grill D (1972) Die Leittähigkeit von Fichtenborken-Extrakien als empfindlicher Indikator für Luftverunreinigungen. Eur $J$ For Pathol 2, 205-215

Landmann G (1991) Dépérissement des forêts et pollution atmosphérique : bilan de 5 années de recherches (1985-1990) dans le cadre du programme DEFORPA. Pollut Atmos 129, 64-69

Legrand I (1991) Végétation lichénique corticole et caractéristiques physico-chimiques des écorces : relations avec la symptomatologie du dépérissement des forêts des Alpes du Nord. Thèse d'Université, Biologie, Université Joseph-Fourier, Grenoble, $225 \mathrm{p}$

Legrand I, Asta J (1991) Lichens épiphytes et caractéristiques physico-chimiques des écorces : relations avec le dépérissement des forêts dans les Alpes du Nord. 116 Congrès national des sociétés savantes, Chambéry, 1991. Sciences, 1-18

Lötschert W, Köhm HJ (1977) Characteristics of tree bark as an indicator in high-emmission areas. CEcologia 27, 47-64

Puech L (1991) Quelques aspects du dépérissement du sapin (Abies alba Mill) dans les Alpes externes dauphinoises : symptomatologie, diagnostic foliaire, pédologie. DEA Écologie, Géographie, Aménagement des montagnes, Université Joseph-Fourier, Grenoble, $83 p+$ annexes

Souchier B (1989) Essai de typologie des symptômes de dépérissement dans les massits dauphinois externes (Vercors, Chartreuse, Belledonne). Journées de Travail DEFORPA, Nancy - Paris (France), février-mars 1989, 1 , 2.2.1-2.2.28 